\title{
Chiral anomalies induced transport in Weyl metals in quantizing magnetic field
}

\author{
Kamal Das, ${ }^{*}$ Sahil Kumar Singh, ${ }^{\dagger}$ and Amit Agarwal $\mathbb{1}^{\dagger}$ \\ Department of Physics, Indian Institute of Technology Kanpur, Kanpur 208016, India
}

(Received 24 March 2020; accepted 10 August 2020; published 29 September 2020)

\begin{abstract}
Weyl metals host relativistic chiral quasiparticles, which display quantum anomalies in the presence of external electromagnetic fields. Here, we study the manifestations of chiral anomalies in the longitudinal and planar magnetotransport coefficients of Weyl metals, in the presence of a quantizing magnetic field. We present a general framework for calculating all the transport coefficients in the regime where multiple Landau levels are occupied. We explicitly show that all the longitudinal and planar transport coefficients show quantum oscillations which are periodic in $1 / B$. Our calculations recover the quadratic- $B$ dependence in the semiclassical regime and predict a linear- $B$ dependence in the ultraquantum limit for all the transport coefficients.
\end{abstract}

DOI: 10.1103/PhysRevResearch.2.033511

\section{INTRODUCTION}

Weyl metals (WMs) host massless chiral relativistic quasiparticles which show very interesting and novel phenomena [1-15]. One of the most interesting aspects of massless relativistic chiral fluids, in quantum field theory is the breakdown of the chiral gauge symmetry in the presence of an external electromagnetic field [16-18]. This results in the chiral anomaly (CA) which manifests in the nonconservation of chiral charge [16-19]. A condensed matter realization of this was first explored in the lattice theory of Weyl fermions by Nielsen and Ninomiya in 1983 [18]. They predicted that the CA will give rise to a positive longitudinal magnetoconductivity, which is linear in the magnetic field strength $(B)$, for ultrahigh magnetic field in the diffusive limit. With the recent realizations of WM [20], there have been several experiments which report positive magnetoconductivity or negative magnetoresistance and attribute it to the CA [1-4,21-24].

Relativistic chiral fluids in a gravitational field also display the mixed chiral-gravitational anomaly, which results in nonconservation of the chiral energy $[25,26]$. This manifests in the magnetothermal experiments in the form of positive magnetothermopower and positive magnetothermal conductivity [26-29], both of which have also been observed in recent experiments $[30,31]$. In addition to their manifestations in longitudinal magnetotransport, CAs have also been shown to give rise to the planar Hall effects in all transport coefficients [29,32-40]. Recently, we predicted another anomaly, the thermal chiral anomaly in which a temperature gradient collinear

\footnotetext{
*kamaldas@iitk.ac.in

†sahilks137@gmail.com

‡amitag@iitk.ac.in
}

Published by the American Physical Society under the terms of the Creative Commons Attribution 4.0 International license. Further distribution of this work must maintain attribution to the author(s) and the published article's title, journal citation, and DOI. with the magnetic field gives rise to charge and energy imbalance between the opposite chirality Weyl fermions [29].

However, the bulk of the theoretical work till date has been focused on the semiclassical transport regime which displays a quadratic- $B$ dependence of all transport coefficients $[6,8,34,41-48]$ or only in the ultraquantum regime [18] (see Fig. 1). Recently, a unified framework for calculating the electrical magnetoconductivity in all the distinct transport regimes have been developed in Refs. [49-51]. So it is natural to ask what happens to the other magnetotransport coefficients. Can all the distinct transport coefficients be explored within a unified framework?

In this paper, we attempt to answer these and other related questions. Here, we generalize the framework of Refs. [50,51] for calculating all the magnetotransport coefficients in the regime where multiple Landau levels are occupied and connect them to the different CAs. We explicitly show that all the longitudinal and planar transport coefficients show quantum oscillations which are periodic in $1 / B$. Our calculations recover the quadratic- $B$ dependence in the semiclassical regime and predict a linear- $B$ dependence in the ultraquantum limit for all the magnetotransport coefficients. The rest of the paper is organized as follows: In Sec. II, we discuss the Landau quantization in WMs, and in Sec. III, we connect the anomalous magnetotransport in WMs to different CAs. In Sec. IV, we present the general formalism of calculating all the CAinduced magnetotransport coefficients. In Sec. V, we calculate the longitudinal magnetotransport coefficients, followed by the exploration of the magnetotransport coefficients in the planar Hall setup in Sec. VI. We discuss the experimental possibilities in Sec. VII and summarize our results in Sec. VIII.

\section{LANDAU QUANTIZATION IN WEYL METAL}

In the presence of a magnetic field, the Hamiltonian of a single Weyl cone (of chirality $s$ ), after Peierls substitution, is given by

$$
\hat{H}^{s}=s v_{F} \boldsymbol{\sigma} \cdot(\hat{\mathbf{p}}+e \mathbf{A}) .
$$



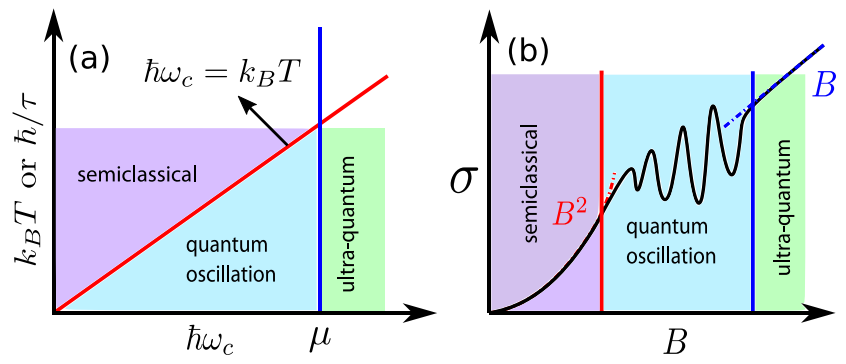

FIG. 1. (a) The three different magnetotransport regimes. In the semiclassical regime (purple area), multiple Landau level are occupied, but the impurity scattering or thermal smearing makes them indistinguishable $\left(\hbar \omega_{c}<k_{B} T\right.$ or $\left.\hbar / \tau\right)$. In the quantum oscillation regime (blue), multiple Landau level are occupied and they are distinguishable, while in the ultraquantum regime (green), only the lowest Landau level is occupied $\left(\hbar \omega_{c}>\mu\right)$. (b) The magnetic field dependence of the magnetoconductance in the three transport regimes for a WM.

Here, $-e$ is the electronic charge, $v_{F}$ is the Fermi velocity, $\boldsymbol{\sigma}=\left(\sigma_{x}, \sigma_{y}, \sigma_{z}\right)$ is a vector composed of the three Pauli matrices, and $\mathbf{A}$ is the vector potential corresponding to the magnetic field $\mathbf{B}=\boldsymbol{\nabla} \times \mathbf{A}$. Considering the magnetic field along the $z$ direction and using the Landau gauge with $\mathbf{A}=$ $(-B y, 0,0)$, it is straightforward to calculate the energy spectrum of the Hamiltonian in Eq. (1). The Landau level (LL) energy spectrum is given by

$$
\epsilon_{n \pm}^{s}= \begin{cases}-s \hbar v_{F} k_{z} & n=0 \\ \pm \sqrt{\left(\hbar v_{F} k_{z}\right)^{2}+2 n\left(\hbar \omega_{c}\right)^{2}} & n \geqslant 1 .\end{cases}
$$

Here, $n$ denotes the LL index, $k_{z}$ is the $z$ component of the crystal momentum, and we have defined the cyclotron frequency, $\omega_{c}=v_{F} / l_{B}$ with $l_{B}=\sqrt{\hbar /(e|B|)}$ being the magnetic length scale. In the rest of the paper, we will use $B=|\mathbf{B}|$. The energy spectrum of Eq. (2) is shown in Figs. 2(a) and 2(b). We emphasize that the lowest LL are chiral in nature, i.e., right (left) movers for negative (positive) chirality node, and will play a crucial role in the CAs [18] discussed in this paper.

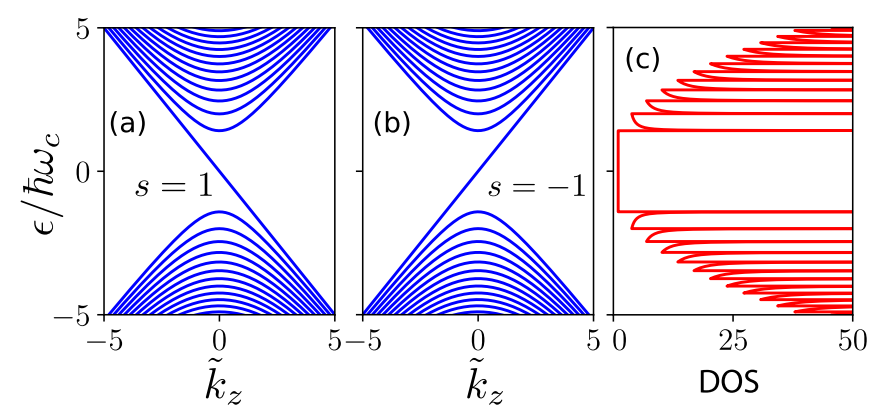

FIG. 2. The Landau level energy spectrum in a WM for (a) positive and (b) negative chirality Weyl node, respectively. The lowest LLs disperse linearly and are chiral in nature. Here, $\tilde{k}_{z} \equiv \hbar v_{F} k_{z} / \mu$. (c) The corresponding density of states (DOS) of the LL spectrum. The DOS is scaled by $\mathfrak{D} /\left(h v_{F}\right)$. The DOS for the lowest $(n=0)$ LL is constant, and it increases as the energy moves away from the Weyl nodes. The oscillation in the DOS will also manifest in all the transport coefficients.
In contrast, all the $n \geqslant 1$ LLs are achiral (support both right and left movers), and they play an important role in quantum oscillations.

Each of the LLs is highly degenerate and the degeneracy is specified by $\mathfrak{D}=1 /\left(2 \pi l_{B}^{2}\right)$. The density of states (DOS) of the LL spectrum is shown in Fig. 2(c). The group velocity of the quasiparticles in these LLs is given by

$$
v_{n z}^{s}=\frac{\partial \epsilon_{n}^{s}}{\hbar \partial k_{z}}= \begin{cases}-s v_{F} & n=0, \\ \hbar v_{F}^{2} k_{z} / \epsilon_{n}^{s} & n \geqslant 1 .\end{cases}
$$

Here, $\epsilon_{n}^{s} \rightarrow \epsilon_{n+}^{s}\left(\epsilon_{n}^{s} \rightarrow \epsilon_{n-}^{s}\right)$ for the positive (negative) energy branches. The carriers of the lowest LLs have a constant velocity, and are either left movers (for $s=1$ ) or right movers (for $s=-1$ ). Next, we discuss the origin of quantum anomalies from the lowest chiral LLs and explore their manifestation in electric and thermal magnetotransport experiments in WM $[25,27,29,41,52]$.

\section{EQUILIBRIUM CURRENTS AND COEFFICIENTS OF CHIRAL ANOMALIES}

One simple way to understand the origin of CAs in $\mathrm{WM}$ is to calculate the equilibrium (no externally applied bias voltage or temperature gradient) current, in the presence of a magnetic field. The equilibrium charge and energy current for each Weyl node is given by $\left\{j_{e, \text { eq }}^{s}, j_{\mathcal{E}, \text { eq }}^{s}\right\}=$ $\mathfrak{D} \sum_{n} \int \frac{d k_{z}}{2 \pi} v_{n z}^{s}\left\{-e, \epsilon_{n}^{s}\right\} f_{n}^{s}$. Here, $f_{n}^{s}$ is the Fermi-Dirac distribution function for the $n$th LL: $f_{n}^{s} \equiv 1 /\left[1+e^{\beta\left(\epsilon_{n}^{s}-\mu\right)}\right]$ with $\beta \equiv 1 / k_{B} T$, and $\mu$ denotes the chemical potential. These expressions for the current are valid for both the $\epsilon_{n+}^{s}$ and the $\epsilon_{n-}^{s}$ branches, which are chosen depending on $\mu$ being positive and negative, respectively. For all the LLs with $n \neq 0$, the integrand is an odd function of $k_{z}$, and thus their contribution to the total charge and energy current is identically zero. Thus, only the $n=0 \mathrm{LL}$ can contribute to the equilibrium charge and energy current. To evaluate this contribution, we will consider the chemical potential to be positive $(\mu>0)$. Evaluating these expressions using integration by parts $[28,29]$, we obtain

$$
\begin{aligned}
& j_{e, \text { eq }}^{s}=-e\left(\mu \mathcal{C}_{0}^{s}+k_{B} T \mathcal{C}_{1}^{s}\right) B, \\
& j_{\mathcal{E}, \text { eq }}^{s}=\left(\mu^{2} \frac{\mathcal{C}_{0}^{s}}{2}+\mu k_{B} T \mathcal{C}_{1}^{s}+k_{B}^{2} T^{2} \frac{\mathcal{C}_{2}^{s}}{2}\right) B .
\end{aligned}
$$

Here, the coefficients $\mathcal{C}_{v}^{s}$ for $v=\{0,1,2\}$ are given by

$$
\mathcal{C}_{v}^{s}=\frac{e}{2 \pi \hbar} \sum_{n} \int \frac{d k_{z}}{2 \pi} v_{n z}^{s}\left(\frac{\epsilon_{n}^{s}-\mu}{k_{B} T}\right)^{v}\left(-\frac{\partial f_{n}^{s}}{\partial \epsilon_{n}^{s}}\right) .
$$

For our choice of $\mu>0$, we have $\epsilon_{n}^{s} \rightarrow \epsilon_{n+}^{s}$. A brief derivation of Eqs. (4) and (5) is sketched in Appendix A. We emphasize here that Eqs. (4)-(6) are exact expressions and these do not require any approximation. In Eq. (6), only the chiral lowest LLs $(n=0)$ contribute to the sum and we have $\mathcal{C}_{v}^{s} \propto s$, the chirality of the Weyl node. Thus, the existence of the chiral LLs is an essential ingredient to obtain the nonzero charge and energy currents for each node even in equilibrium. These chiral currents are in turn related to the CAs in WMs [27,29,52]. We had earlier derived equations similar to Eqs. (4) and (5) in the semiclassical regime, where the Berry curvature of the Weyl nodes played an important role [29]. 


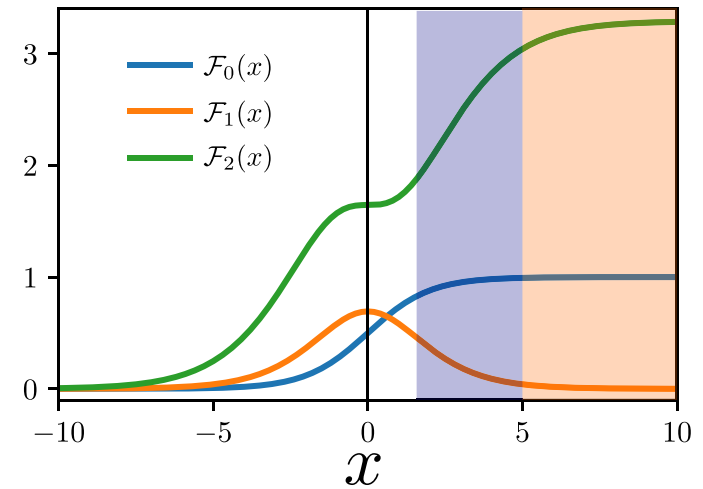

FIG. 3. The temperature dependence $(x=\beta \mu)$ of the dimensionless functions associated with the CAs. Note that in $x \rightarrow \infty$ or $T \rightarrow 0$ for a finite $\mu>0$ limit, these functions are constant as shown in the orange shaded region. In this region, the coefficient of thermal chiral anomaly $\mathcal{F}_{1} \rightarrow 0$. However, it has a finite value in the finite $x$ regime. For this paper, we will mostly focus on the regime $\mu>k_{B} T$ or $x>1$, which is shaded in blue, and on the $\mu \gg k_{B} T$ regime, shaded in orange.

The quantities $\mathcal{C}_{0}^{s}, \mathcal{C}_{2}^{s}$, and $\mathcal{C}_{1}^{s}$ are known as the coefficient of the chiral (or axial) anomaly [41], the coefficient of the mixed chiral (or axial) gravitational anomaly $[25,27,52]$ and the coefficient of the thermal chiral (or axial) anomaly [29], respectively. We emphasize that the coefficient $\mathcal{C}_{1}^{s}$ vanishes in the $\beta \mu \rightarrow \infty$ limit and has been relatively unexplored. Evaluating Eq. (6), we obtain

$$
\mathcal{C}_{v}^{s}=-s \frac{e}{4 \pi^{2} \hbar^{2}} \begin{cases}\mathcal{F}_{0}(\beta \mu) & v=0, \\ \mathcal{F}_{1}(\beta \mu) & v=1, \\ \mathcal{F}_{2}(\beta \mu) & v=2 .\end{cases}
$$

Here, we have defined $\mathcal{F}_{0}(x)=\frac{1}{1+e^{-x}}$ for the chiral anomaly, $\mathcal{F}_{1}(x)=\frac{x}{1+e^{x}}+\ln \left(1+e^{-x}\right)$ for the thermal chiral anomaly, and $\mathcal{F}_{2}(x)=\frac{\pi^{2}}{3}+2 \operatorname{Li}_{2}\left(-e^{-x}\right)-2 x \ln \left(1+e^{-x}\right)-\frac{x^{2}}{1+e^{x}}$ for the mixed chiral-gravitational anomaly. The $x$ dependence of these functions is explicitly shown in Fig. 3. In the $x \rightarrow \infty$ limit, these functions are constant [see Eq. (20)], with $\mathcal{F}_{2}$ being the largest of the three and $\mathcal{F}_{1} \rightarrow 0$. However, the thermal chiral anomaly coefficient $\mathcal{F}_{1}$ has a finite value for finite $x$.

In equilibrium, the total charge and energy current from all the Weyl nodes in a WM adds up to zero, as opposite chirality nodes always appear in pairs in a WM. However, the chiral charge current $\left(j_{e, \text { eq }}^{+}-j_{e \text { eq }}^{-}\right)$and energy current $\left(j_{\mathcal{E} \text {, eq }}^{+}-\right.$ $\left.j_{\mathcal{E}, \text { eq }}^{-}\right)$are nonzero even in equilibrium. More interestingly, in the presence of an external electric field or a temperature gradient, this leads to charge and energy imbalance between pair of opposite chirality Weyl nodes. Below, we explore the consequence of this in magnetotransport experiments.

\section{NONEQUILIBRIUM CURRENTS AND CHIRAL ANOMALIES}

An applied electric field or temperature gradient drives the system out of equilibrium. In the Boltzmann transport formalism, the nonequilibrium distribution function (NDF), $g_{n}^{s}$, within the relaxation time approximation, satisfies the following equation $[29,50]$ :

$$
\partial_{t} g_{n}^{s}+\dot{\mathbf{k}}_{n}^{s} \cdot \nabla_{\mathbf{k}} g_{n}^{s}+\dot{\mathbf{r}}_{n}^{s} \cdot \nabla_{\mathbf{r}} g_{n}^{s}=-\frac{g_{n}^{s}-\bar{g}_{n}^{s}}{\tau}-\frac{\bar{g}_{n}^{s}-f_{n}^{s}}{\tau_{v}} .
$$

Here, $\bar{g}_{n}^{s}$ is the local equilibrium distribution function considered to be the Fermi function for the $n$th LL with a nodedependent chemical potential $\mu^{s}$ and temperature $T^{s}$. The first term in the right-hand side of Eq. (8) represents the relaxation of the NDF to the local equilibrium through the intranode scattering rate $1 / \tau$. The intranode scattering does not alter the number of carriers in the respective node, and its impact is similar to that in other metals as well. In contrast, the second term represents the internode scattering with a relaxation rate of $1 / \tau_{v}$, which attempts to undo the impact of the chiral imbalance [Eqs. (9) and (10)] and restore a steady-state carrier distribution function. In a typical WM with broken time-reversal symmetry, the Weyl nodes are separated in the momentum space. If we are in a regime of small $\mu$ so that the Fermi wave vector is smaller than the separation of the Weyl nodes, then we have $\tau_{v} \gg \tau$ [8].

The idea of a steady state in the presence of CAs and internode scattering becomes more evident from the continuity equations of particle number and heat density for electric field and temperature gradient applied along the direction of magnetic field. Integrating Eq. (8) over all the states in a single cone, we obtain the particle number conservation equation (within the linear response) to be

$$
\frac{\partial \mathcal{N}^{s}}{\partial t}+\nabla_{\mathbf{r}} \cdot \mathbf{J}_{n}^{s}+e E B \mathcal{C}_{0}^{s}=-\frac{\mathcal{N}^{s}-\mathcal{N}_{0}^{s}}{\tau_{v}} .
$$

Here, $\nabla_{\mathbf{r}} \cdot \mathbf{J}_{n}^{s}=k_{B} \mathcal{C}_{1}^{s} B \nabla T$ is the divergence of the particle current. The quantities $\left\{\mathcal{N}_{0}^{s}, \mathcal{N}^{s}\right\}=\mathfrak{D} \sum_{n} \int \frac{d k_{z}}{2 \pi}\left\{f_{n}^{s}, g_{n}^{s}\right\}$ are the total particle number density in each Weyl cone before and after applying external fields, respectively. In Eq. (9), the terms with $\mathcal{C}_{0}^{s} E B$ and $\mathcal{C}_{1}^{s} B \nabla T$ represent CA-induced flow of particle [29].

Following a similar procedure, we construct the continuity equation for heat density. Multiplying Eq. (8) by $\tilde{\epsilon}_{n}^{s} \equiv \epsilon_{n}^{s}-\mu$ and integrating over all the states of a given Weyl node, we obtain

$$
\frac{\partial \mathcal{Q}^{s}}{\partial t}+\nabla_{\mathbf{r}} \cdot \mathbf{J}_{Q}^{s}+e E B \mathcal{C}_{1}^{s} k_{B} T=-\frac{\mathcal{Q}^{s}-\mathcal{Q}_{0}^{s}}{\tau_{v}} .
$$

Here, $\nabla_{\mathbf{r}} \cdot \mathbf{J}_{Q}^{s}=k_{B}^{2} \mathcal{C}_{2}^{s} T B \nabla T$ is the divergence of the heat current. The quantities $\left\{\mathcal{Q}_{0}^{s}, \mathcal{Q}^{s}\right\}=\mathfrak{D} \sum_{n} \int \frac{d k_{z}}{2 \pi} \tilde{\epsilon}_{n}^{s}\left\{f_{n}^{s}, g_{n}^{s}\right\}$ are the heat density in each cone, before and after applying external fields, respectively. In Eq. (10), $E B \mathcal{C}_{1}^{s}$ and $\mathcal{C}_{2}^{s} B \nabla T$ represent the CA-induced flow of the heat density [29]. In constructing Eqs. (9) and (10), we have used the fact that the intranode scattering does not change the number of carrier and energy of each Weyl node.

To evaluate the charge and heat currents in this steady state, we now calculate the NDF from Eq. (8). Within the linear response regime, it is reasonable to expect that the change in chemical potential and temperature in each cone is small: $\delta \mu^{s} \equiv \mu^{s}-\mu<\mu$ and $\delta T^{s} \equiv T^{s}-T<T$. Then to 

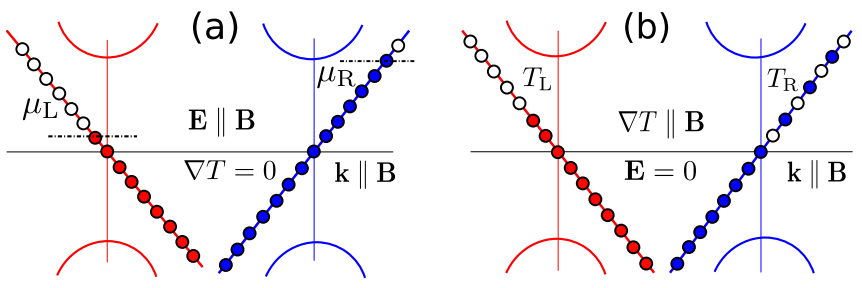

FIG. 4. Chiral anomalies induced charge and energy imbalance in a WM in the ultraquantum limit. (a) The electrical chiral anomaly induced nonconservation of chiral charge in the presence of parallel electric and magnetic fields. (b) The mixed chiral-gravitational anomaly induced nonconservation of chiral energy in the presence of a temperature gradient parallel to the magnetic field.

the lowest order in $\delta \mu^{s}$ and $\delta T^{s}$, the NDF can be expressed as

$$
\begin{aligned}
g_{n}^{s}= & f_{n}^{s}-\tau v_{n z}^{s}\left(e E+\frac{\epsilon_{n}^{s}-\mu}{T} \nabla T\right)\left(-\frac{\partial f_{n}^{s}}{\partial \epsilon_{n}^{s}}\right) \\
& +\left(1-\frac{\tau}{\tau_{v}}\right)\left(\delta \mu^{s}+\frac{\epsilon_{n}^{s}-\mu}{T} \delta T^{s}\right)\left(-\frac{\partial f_{n}^{s}}{\partial \epsilon_{n}^{s}}\right) .
\end{aligned}
$$

In Eq. (11), the chiral chemical potential $\delta \mu^{s}$ and chiral temperature $\delta T^{s}$ are given by

$$
\left(\begin{array}{c}
\delta \mu^{s} \\
k_{B} \delta T^{s}
\end{array}\right)=-\tau_{v} B\left[\mathcal{D}^{s}\right]^{-1}\left(\begin{array}{cc}
\mathcal{C}_{0}^{s} & \mathcal{C}_{1}^{s} \\
\mathcal{C}_{1}^{s} & \mathcal{C}_{2}^{s}
\end{array}\right)\left(\begin{array}{c}
e E \\
k_{B} \nabla T
\end{array}\right)
$$

Here, we have defined the magnetic-field-dependent generalized energy density matrix, $\mathcal{D}^{s} \equiv\left(\begin{array}{ll}\mathcal{D}_{0}^{s} & \mathcal{D}_{1}^{s} \\ \mathcal{D}_{1}^{s} & \mathcal{D}_{2}^{s}\end{array}\right)$, with

$$
\mathcal{D}_{\nu}^{s}=\mathfrak{D} \sum_{n} \int \frac{d k_{z}}{2 \pi}\left(\frac{\epsilon_{n}^{s}-\mu}{k_{B} T}\right)^{v}\left(-\frac{\partial f_{n}^{s}}{\partial \epsilon_{n}^{s}}\right)
$$

and $v=\{0,1,2\}$.

Equation (12) quantifies the chiral charge and energy imbalance in the two Weyl nodes. These imbalances are proportional to the coefficients of CAs and inversely proportional to the generalized energy density. The former is due to the fact that CAs are responsible for the charge and heat imbalances. The latter is a consequence of the fact that a smaller DOS will lead to a larger change in $\delta \mu^{s}$ and $\delta T^{s}$ and vice versa. These imbalances are schematically depicted in Fig. 4 in the ultraquantum limit with only the lowest LLs being occupied. Figure 4(a) shows the $\delta \mu^{s}$ induced by the electrical chiral anomaly and Fig. 4(b) displays the $\delta T^{s}$ generated by the mixed chiral-gravitational anomaly. In addition to these two anomalies, the thermal chiral anomaly has a nonzero contribution to both the charge and energy imbalances, though this contribution is relatively smaller [29].

\section{LONGITUDINAL MAGNETOTRANSPORT}

Having obtained the NDF in the Landau quantization regime, we now proceed to calculate the magnetotransport coefficients. The steady-state nonequilibrium charge and heat current for each Weyl node is defined as $\left\{j_{e}^{s}, j_{Q}^{s}\right\}=$ $\sum_{n} \mathfrak{D} \int \frac{d k_{z}}{2 \pi} v_{n z}^{s}\left\{-e, \tilde{\epsilon}_{n}^{s}\right\} g_{n}^{s}$. Focusing only on the anomalyinduced contribution which is proportional to the internode scattering time $\tau_{v}$, we obtain

$$
\begin{aligned}
\left(\begin{array}{c}
j_{e}^{s} \\
j_{Q}^{s}
\end{array}\right)= & -\tau_{v} B^{2}\left(\begin{array}{cc}
-e \mathcal{C}_{0}^{s} & -e \mathcal{C}_{1}^{s} \\
k_{B} T \mathcal{C}_{1}^{s} & k_{B} T \mathcal{C}_{2}^{s}
\end{array}\right)\left[\mathcal{D}^{s}\right]^{-1}\left(\begin{array}{cc}
\mathcal{C}_{0}^{s} & \mathcal{C}_{1}^{s} \\
\mathcal{C}_{1}^{s} & \mathcal{C}_{2}^{s}
\end{array}\right) \\
& \times\left(\begin{array}{c}
e E \\
k_{B} \nabla T
\end{array}\right)
\end{aligned}
$$

Note that in Eq. (14), the magnetic field dependence of the charge and heat current comes from (i) the $B^{2}$ term, (ii) the DOS which depends on $B$ via the LL spectrum, and (iii) magnetic field dependence of $\tau_{v}$. The transport coefficients can now be easily obtained from the phenomenological relations for linear response: $j_{e, i}=\sum_{j}\left[\sigma_{i j} E_{j}-\alpha_{i j} \nabla_{j} T\right]$ and $j_{Q, i}=\sum_{j}\left[\bar{\alpha}_{i j} E_{j}-\bar{\kappa}_{i j} \nabla_{j} T\right]$. Here, $\sigma, \alpha, \bar{\alpha}$, and $\bar{\kappa}$ denote the electrical, thermoelectric, electrothermal, and constant voltage thermal conductivity matrix, respectively. In the limiting case of $\beta \mu \rightarrow \infty$ (or $\mu \gg k_{B} T$ ), the thermal chiral anomaly coefficient $\mathcal{C}_{1}^{s} \rightarrow 0$. In the same limit, we find $\mathcal{D}_{0}^{s}, \mathcal{D}_{2}^{s} \gg \mathcal{D}_{1}^{s}$. Using these, Eq. (14) can be rewritten as

$$
\left(\begin{array}{l}
j_{e}^{s} \\
j_{Q}^{s}
\end{array}\right)=\tau_{v} B^{2}\left(\begin{array}{cc}
\frac{1}{\mathcal{D}_{0}^{s}}\left(e \mathcal{C}_{0}^{s}\right)^{2} & \frac{\mathcal{D}_{1}^{s}}{\mathcal{D}_{0}^{s} \mathcal{D}_{2}^{s}} e \mathcal{C}_{0}^{s} k_{B} \mathcal{C}_{2}^{s} \\
T \frac{\mathcal{D}_{1}^{s}}{\mathcal{D}_{0}^{s} \mathcal{D}_{2}^{s}} e \mathcal{C}_{0}^{s} k_{B} \mathcal{C}_{2}^{s} & T \frac{1}{\mathcal{D}_{2}^{s}}\left(k_{B} \mathcal{C}_{2}^{s}\right)^{2}
\end{array}\right)\left(\begin{array}{c}
E \\
-\nabla T
\end{array}\right) .
$$

From Eq. (15), we note that $\sigma \propto\left(\mathcal{C}_{0}^{s}\right)^{2}, \bar{\kappa} \propto\left(\mathcal{C}_{2}^{s}\right)^{2}$, and $\alpha \propto$ $\bar{\alpha} \propto \mathcal{C}_{0}^{s} \mathcal{C}_{2}^{s}$. Thus, it is reasonable to associate different transport coefficients with different CAs. The presence of thermal chiral anomaly coefficient in the more general Eq. (14) leads to a more complicated dependence of the transport coefficients on the anomaly coefficients.

We now explore the different regimes of magnetotransport depending on the strength of applied magnetic field: (i) the ultraquantum regime where only the lowest $(n=0)$ LLs are occupied, (ii) the quantum oscillation regime with a few distinguishable LLs being occupied, and (iii) the semiclassical regime with many but indistinguishable LLs being occupied.

\section{A. Ultraquantum regime}

In the ultraquantum regime, only the lowest $(n=0) \mathrm{LL}$ is occupied and we have $\hbar \omega_{c} \gg \mu$. In this regime, the CA coefficients have already been calculated in Eq. (7). We calculate the finite-temperature DOS and its energy moments defined in Eq. (13) to be

$$
\mathcal{D}_{\nu, 0}^{s}=\frac{\mathfrak{D}}{2 \pi \hbar v_{F}} \begin{cases}\mathcal{F}_{0}(\beta \mu) & v=0, \\ \mathcal{F}_{1}(\beta \mu) & v=1 \\ \mathcal{F}_{2}(\beta \mu) & v=2\end{cases}
$$

Note that similar to the coefficient of thermal CA, $\mathcal{C}_{1}^{s}$, the first energy moment of the DOS, $\mathcal{D}_{1,0}^{s}$ is relatively smaller than the other two. Using these expressions in Eq. (14), we obtain the anomaly-induced charge current to be

$$
j_{e}^{s}=\frac{e}{h} \frac{e B \tau_{v} v_{F}}{h}\left[e \mathcal{F}_{0}(\beta \mu) E+k_{B} \mathcal{F}_{1}(\beta \mu) \nabla T\right] .
$$


We highlight the linear- $B$ dependence of the charge current. The corresponding charge conductivity is given by $\sigma_{z z}^{s}=\frac{e^{2}}{4 \pi^{2} \hbar} \frac{v_{F} \tau_{v}}{l_{B}^{2}} \mathcal{F}_{0}(\beta \mu)$. Since $\mathcal{F}_{0}(x \rightarrow \infty) \approx 1$, this reproduces the previously obtained results $[18,41,49,53,54]$ in the limit $\beta \mu \rightarrow \infty$.

The corresponding thermoelectric conductivity is given by $\alpha_{z z}^{s}=-\frac{k_{B} e}{4 \pi^{2} \hbar} \frac{v_{F} \tau_{v}}{l_{B}^{2}} \mathcal{F}_{1}(\beta \mu)$ which is small but nonzero for finite $\beta \mu$. Remarkably, we find that the magnetic-field-induced thermoelectric conductivity is negative, unlike its semiclassical counterpart $[29,47]$ and violates the Mott relation. However, in the limiting case of $\beta \mu \rightarrow \infty, \alpha_{z z}^{s} \rightarrow 0$, consistent with the results of Refs. [27,52]. As a consequence of this vanishing $\alpha$, the magnetic-field-induced change in the Seebeck coefficient, $S=\alpha \sigma^{-1}$, is equal to the magnetic-field-induced change in $\sigma$. This in turn implies that the magnetoresistance (MR) in the Seebeck coefficient is equal to the MR in the resistivity. This is in contrast with the semiclassical regime where the MR in the Seebeck coefficient is twice that of the MR in the resistivity $[29,31,47]$.

The CA-induced heat current is obtained to be

$$
j_{Q}^{s}=\frac{k_{B} T}{h} \frac{e B v_{F} \tau_{v}}{h}\left[-e \mathcal{F}_{1}(\beta \mu) E-k_{B} \mathcal{F}_{2}(\beta \mu) \nabla T\right] .
$$

Similar to the charge current, the heat current also shows a linear- $B$ dependence. The validity of the Onsager's reciprocity relation, $\bar{\alpha}(\mathbf{B})=T \alpha(-\mathbf{B})$ can also be confirmed. The constant voltage thermal conductivity is given by

$$
\bar{\kappa}_{z z}^{s}=\frac{k_{B}^{2} T}{4 \pi^{2} \hbar} \frac{v_{F} \tau_{v}}{l_{B}^{2}} \mathcal{F}_{2}(\beta \mu) .
$$

Interestingly, for finite $\beta \mu$, we find that $\bar{\kappa}_{z z}^{s}$ and $\sigma_{z z}^{s}$ are not connected via the Wiedemann-Franz law. However, the Wiedemann-Franz law gets restored in the $\beta \mu \rightarrow \infty$ limit, and we have $\bar{\kappa}_{z z}^{s}=\frac{\pi^{2}}{3}\left(\frac{k_{B}}{e}\right)^{2} \sigma_{z z}^{s}$. This linear- $B$ dependence of the thermal conductivity in the ultraquantum limit has also been observed in recent experiments [52,55].

\section{B. Quantum oscillation regime}

In this section, we will discuss the scenario when multiple LLs are occupied. For this, the chemical potential has to be larger than the separation between the LLs $\left(\mu>\hbar \omega_{c}\right)$. Since we want to highlight the oscillations in the magnetotransport coefficients, we work in the regime $k_{B} T \ll \hbar \omega_{c}$, so that the temperature broadening does not smear out the signatures of the discrete LLs. These two conditions combine to yield $\mu \gg k_{B} T$ and thus we focus on the limit of $\beta \mu \rightarrow \infty$ in this section. In this limit, the coefficients of CAs are given by

$$
\mathcal{C}_{\nu}^{s}=-s \frac{e}{4 \pi^{2} \hbar^{2}} \begin{cases}1 & v=0, \\ 0 & v=1, \\ \pi^{2} / 3 & v=2 .\end{cases}
$$

In contrast to the anomaly coefficients, the DOS and its energy moments get contributions from all the filled LLs.
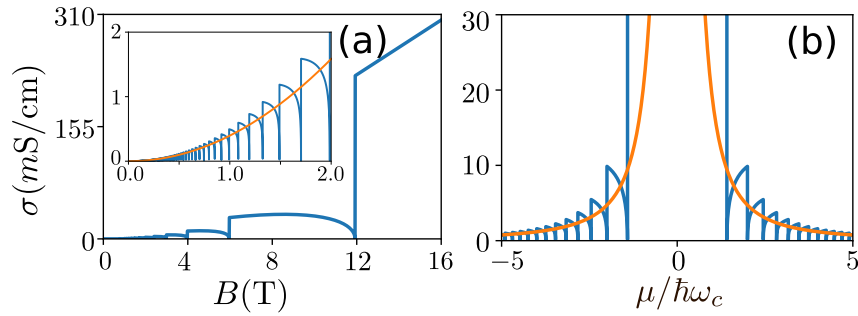

FIG. 5. (a) The variation of the longitudinal charge conductivity with $B$ (for $\mu=25 \mathrm{meV}$ ). Note the linear- $B$ dependence in the ultraquantum regime. The inset shows the low-field behavior and the semiclassical quadratic- $B$ dependence is shown in orange. (b) The $\mu$ dependence of the longitudinal charge conductivity (for $B=2 \mathrm{~T}$ ). Note that the spurious divergence of the longitudinal conductivity as $\mu \rightarrow 0$ in the semiclassical limit is not there in the LL picture. Here, we have chosen $v_{F}=2 \times 10^{5} \mathrm{~m} / \mathrm{s}$ and $\tau_{v}=10^{-9} \mathrm{~s}$.

The highest occupied LL index can be calculated to be $n_{c}=$ $\operatorname{int}\left[\mu^{2} /\left(2 \hbar^{2} \omega_{c}^{2}\right)\right]$, and we obtain

$$
\mathcal{D}_{v}^{s}=\frac{\mathfrak{D}}{2 \pi \hbar v_{F}} \begin{cases}\Theta_{0} & v=0, \\ \frac{2 \pi^{2}}{3 \beta \mu} \Theta_{1} & v=1, \\ \frac{\pi^{2}}{3} \Theta_{0} & v=2 .\end{cases}
$$

Here, we have defined $\Theta_{0} \equiv 1+\sum_{n=1}^{n_{c}} 2 / \lambda_{n}$ and $\Theta_{1} \equiv$ $-\sum_{n=1}^{n_{c}} 2 n\left(\hbar \omega_{c} / \mu\right)^{2} / \lambda_{n}^{3}$ with $\lambda_{n}=\sqrt{1-2 n\left(\hbar \omega_{c} / \mu\right)^{2}}$. Note that $n_{c} \propto$ int $[1 / B]$. So the number of occupied LLs is inversely proportional to $B$. This combined with Eq. (21a) is what leads to quantum oscillations in the longitudinal magnetoconductivity with a period proportional to $1 / B$. As a consistency check, we note that Eq. (21a) obtained here, is identical to Eq. (4) of Ref. [50]. We find that while $\mathcal{D}_{0}^{s}$ and $\mathcal{D}_{2}^{s}$ are more or less temperature independent (for $\mu>k_{B} T$ ), $\mathcal{D}_{1}^{s}$ depends inversely on $\beta \mu$ and vanishes in the limit $T \rightarrow 0$. Using Eqs. (20) and (21) in Eq. (15), we calculate the charge current to be

$$
j_{e}^{s}=\frac{e^{2} B \tau_{v} v_{F}}{h^{2}}\left(\frac{e}{\Theta_{0}} E-k_{B} \frac{2 \pi^{2}}{3 \beta \mu} \frac{\Theta_{1}}{\Theta_{0}^{2}} \nabla T\right) .
$$

The $1 / \Theta_{0}$ term in the charge conductivity originates from the $1 / \mathcal{D}_{0}^{s}$ term in Eq. (15), and it gives rise to oscillations in the longitudinal conductivity which are periodic in $1 / B$ $[49,50]$. The $B$ dependence of the longitudinal conductivity is displayed in Fig. 5. Additionally, we find that the longitudinal thermoelectric conductivity also shows quantum oscillations arising from the discreteness of the LLs. In this case, the $\Theta_{1} / \Theta_{0}^{2}$ term in Eq. (22) governs the features of the quantum oscillations. The Onsager's reciprocity relation, $\bar{\alpha}(\mathbf{B})=T \alpha(-\mathbf{B})$, holds for the longitudinal thermoelectric conductivity, even in the quantum oscillation regime.

We calculate the heat current to be

$$
j_{Q}^{s}=k_{B} T \frac{e B \tau_{v} v_{F}}{h^{2}} \frac{\pi^{2}}{3}\left(\frac{2 e}{\beta \mu} \frac{\Theta_{1}}{\Theta_{0}^{2}} E-\frac{k_{B}}{\Theta_{0}} \nabla T\right) .
$$

Equations (22) and (23) are the main results of this paper. We have demonstrated that the longitudinal thermal conductivity 


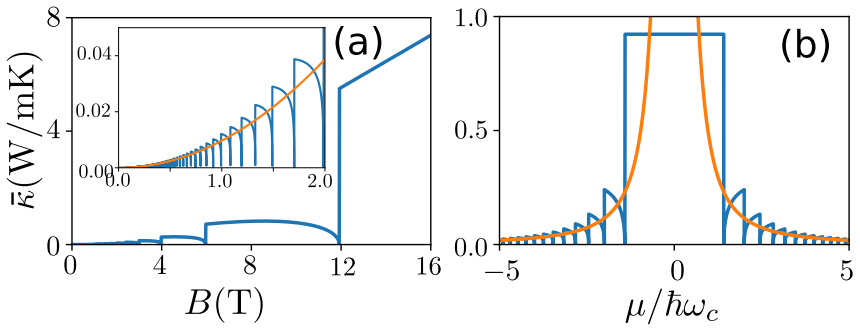

FIG. 6. (a) The variation of the longitudinal thermal conductivity with $B$. The linear- $B$ dependence in the ultraquantum regime (for $B>$ $12 \mathrm{~T}$ here) is evident. The inset shows the small $B$ behavior and the semiclassical $B^{2}$ dependence is shown by the orange line. (b) The $\mu$ dependence of the longitudinal thermal conductivity. The parameters are identical to those of Fig. 5 and $T=10 \mathrm{~K}$.

also possess quantum oscillations with features very similar to that of the charge conductivity (both dictated by $1 / \Theta_{0}$ ) [56]. The dependence of the thermal conductivity on the magnetic field and the chemical potential is shown in Fig. 6.

Remarkably, we find that the period of oscillations of the longitudinal transport coefficients $\sigma$ and $\bar{\kappa}$, defined in Eqs. (22) and (23), satisfies the Onsager's quantization rule for Shubnikov-de Haas oscillations [57] in the transverse conductivity. However, this is not surprising, considering the fact that for both of these the origin lies in the DOS of the LLs. Onsager's quantization rule for the Shubnikov-de Haas oscillations in the transverse conductivity states that the period of conductivity oscillation (in $1 / B$ ) is given by

$$
\Delta\left(\frac{1}{B}\right)=\frac{2 \pi e}{\hbar} \frac{1}{A_{e}} .
$$

Here, $A_{e}$ is the extremal cross section of the Fermi surface in a plane perpendicular to the magnetic field. For our longitudinal conductivity, we find that both the charge and thermal conductivity vanishes at $\mu^{2}=2 n\left(\hbar \omega_{c}\right)^{2}$. This yields the period of oscillation to be $\Delta_{1}(1 / B)=2 e \hbar v_{F}^{2} / \mu^{2}$. Since the Fermi surface in an isotropic WM is spherical with $A_{e}=\pi k_{F}^{2}, \Delta_{1}(1 / B)$ is consistent with Eq. (24). The quantum oscillation in $1 / B$ in the longitudinal components of $\sigma$ and $\bar{\kappa}$ are explicitly shown in Fig. 7.

\section{Semiclassical regime}

In this section, we show that for small $B$ when many LLs are occupied, we can recover the semiclassical results for the

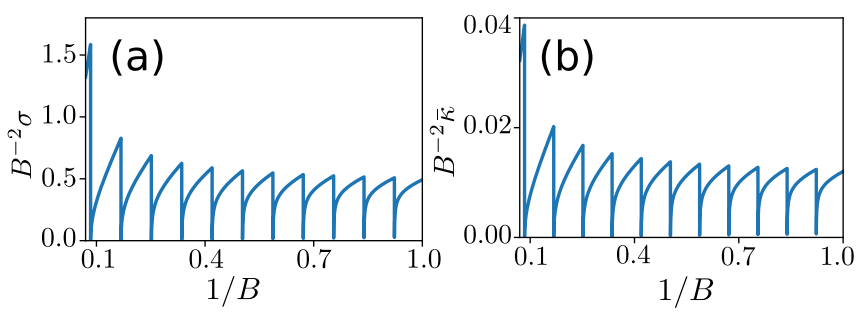

FIG. 7. The (a) charge and (b) thermal conductivity as a function of the inverse of the magnetic field. The constant period of oscillation in $1 / B$ is evident. The period of oscillation is determined by Onsager's quantization rule defined in Eq. (24).
CA-induced transport coefficients [29]. For closely spaced LLs such that $\mu \gg \hbar \omega_{c}$, we can replace the discrete sum over LLs by an integral: $\sum_{0}^{n_{c}} \rightarrow \int_{0}^{n_{c}} d n$. In this limit, we obtain $\Theta_{0} \approx 2\left(\mu / \hbar \omega_{c}\right)^{2}$ and $\Theta_{1} \approx 2\left(\mu / \hbar \omega_{c}\right)^{2}$. Using these expressions, it is straightforward to calculate

$$
\mathcal{D}_{v}^{s} \approx \frac{\mu^{2}}{2 \pi^{2}} \frac{1}{\hbar^{3} v_{F}^{3}} \begin{cases}1 & v=0, \\ \frac{2 \pi^{2}}{3 \beta \mu} & v=1, \\ \frac{\pi^{2}}{3} & v=2 .\end{cases}
$$

These expressions are identical to the DOS derived in Ref. [29].

Now, the charge current can be obtained to be

$$
j_{e}^{s}=\frac{e^{2}}{8 \pi^{2} \hbar} \frac{(e B)^{2} v_{F}^{2}}{\mu^{2}} \tau_{v} v_{F}\left(E-\frac{k_{B}}{e} \frac{2 \pi^{2}}{3 \mu \beta} \nabla T\right) .
$$

The charge conductivity is identical to the previously reported results $[29,41,47]$ which show quadratic- $B$ and positive magnetoconductivity. This semiclassical quadratic- $B$ dependence is a well-established signature of the CA in the low-field limit, and it has also been experimentally verified [21-23]. The thermoelectric conductivity is also consistent with the previously obtained semiclassical results $[29,47]$, and with the experimental observations in Dirac metals and/or semimetals [30,31]. A similar calculation yields the heat current

$$
j_{Q}^{s}=\frac{k_{B} T}{8 \pi^{2} \hbar} \frac{(e B)^{2} v_{F}^{2}}{\mu^{2}} \tau_{v} v_{F} \frac{\pi^{2}}{3}\left(\frac{2 e}{\beta \mu} E-k_{B} \nabla T\right) .
$$

We note that the thermal conductivity obtained here is identical to the previous reports $[29,47]$ and it has recently been measured in GdPtBi [52]. The magnetic field and the Fermi energy dependence of charge conductivity in this regime are shown in Fig. 5 on top of the quantized LL results. In Fig. 5(a), the three different transport regimes, with different $B$ dependences, are evident. In the inset, the yellow line shows the semiclassical fitting. In Fig. 5(b), the Fermi energy dependence is displayed. In Fig. 6, we have shown the same for the thermal conductivity.

\section{PLANAR HALl EFFECTS}

So far we have explored the impact of CAs in the longitudinal magnetotransport coefficients. However, it has been shown that the origin of planar Hall effects and anisotropic longitudinal transport coefficients in nonmagnetic materials can also be related to CAs [32-36]. Here, we explore the impact of quantized LLs, on all the planar Hall transport coefficients and explore the possibility of quantum oscillations in them.

In the planar Hall setup [37-40], we measure the longitudinal and Hall transport coefficients in the plane of the $\mathbf{E}-\mathbf{B}$ (or $\nabla T-\mathbf{B}$ ) fields, as shown in Fig. 8(a). Here the electric field is applied along the $z$ direction with a planar magnetic field applied at an angle $\phi$, so that $\mathbf{B}=B(\cos \phi \hat{\mathbf{z}}+\sin \phi \hat{\mathbf{y}})$. However, it turns out that the calculations for the case where the electric field or temperature gradient is parallel or perpendicular to the magnetic field are relatively easier [58,59]. Thus, we perform our calculations in a rotated frame of reference $\left(z^{\prime}-y^{\prime}\right)$, so that the magnetic field lies along the $z^{\prime}$ axis of the new frame, as 


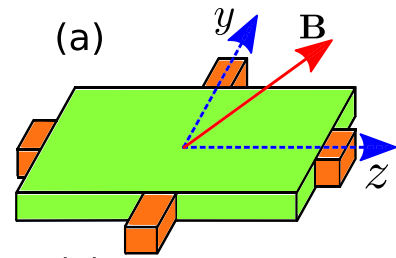

(c)

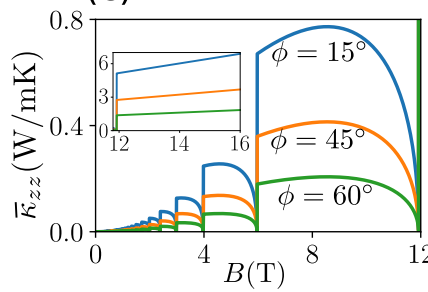

FIG. 8. (a) A schematic of the planar Hall geometry experiments. (b) For ease of calculations, we work in the frame of reference with an axis aligned along the magnetic field. This is rotated with respect to the laboratory frame (aligned along the electric field or temperature gradient) by an angle $\phi$. The $B$ dependence of (a) the anisotropic longitudinal thermal conductivity $\left(\bar{\kappa}_{z z} \propto \cos ^{2} \phi\right)$ and (b) the planar Righi-Leduc effect $\left(\bar{\kappa}_{y z} \propto \cos \phi \sin \phi\right)$ for different angles between the magnetic field and the temperature gradient. The inset shows the contribution of the lowest LL.

shown in Fig. 8(b). The coordinates in the two frames are related as

$$
\left(\begin{array}{l}
z^{\prime} \\
y^{\prime}
\end{array}\right)=\left(\begin{array}{cc}
\cos \phi & \sin \phi \\
-\sin \phi & \cos \phi
\end{array}\right)\left(\begin{array}{l}
z \\
y
\end{array}\right)
$$

If the transport coefficients are denoted by $L_{i j}^{\prime}$ in this rotated frame, then the transport coefficients in the laboratory frame, $L_{i j}=\left\{\sigma_{i j}, \alpha_{i j}, \bar{\alpha}_{i j}, \bar{\kappa}_{i j}\right\}$, are given by

$$
\begin{aligned}
\left(\begin{array}{ll}
L_{z z} & L_{z y} \\
L_{y z} & L_{y y}
\end{array}\right)= & \left(\begin{array}{cc}
\cos \phi & -\sin \phi \\
\sin \phi & \cos \phi
\end{array}\right)\left(\begin{array}{ll}
L_{z z}^{\prime} & L_{z y}^{\prime} \\
L_{y z}^{\prime} & L_{y y}^{\prime}
\end{array}\right) \\
& \times\left(\begin{array}{cc}
\cos \phi & \sin \phi \\
-\sin \phi & \cos \phi
\end{array}\right) .
\end{aligned}
$$

For an electric field applied at an angle to the magnetic field, there are two different effects at play. The parallel component of the electric field (the component parallel to the magnetic field: $E_{\|}=E \cos \phi$ ) makes the crystal momentum time dependent: $k_{z}^{\prime} \rightarrow k_{z}+e E_{\|} t / \hbar$. This modifies the NDF of the electronic states as shown in Eq. (11), with the substitution $E \rightarrow E_{\|}$. The perpendicular component of the electric field $\left(E_{\perp}=E \sin \phi\right)$ modifies the LL spectrum and the associated DOS, as derived in detail in Appendix B. The modified LL spectrum gives rise to a nonlinear (in $E_{\perp}$ ) DOS as shown in Eq. (B9). A similar approach was used in Ref. [51] to demonstrate that the longitudinal planar conductivity has a $\cos ^{6} \phi$ angular dependence, and the planar Hall component has a $\cos ^{5} \phi \sin \phi$ angular dependence, arising from the nonlinear terms in the DOS. However, in this work we work in the linear response regime and focus on the quantum oscillation of the planar thermal transport coefficients. Using the LL dispersion, we calculate the velocity along the magnetic field (to zeroth order in $E_{\perp}$ ) to be

$$
v_{n z^{\prime}}=v_{F}\left(s_{n} \frac{k_{z}}{\sqrt{\frac{2 n}{\tilde{i}_{B}^{2}}+k_{z}^{2}}}-s \delta_{n, 0}\right) .
$$

Here, $s_{n}=\operatorname{sgn}(n)$. There is also a Lorentz velocity component along the $x$ direction (perpendicular to the $\mathbf{B}-\mathbf{E}$ plane), $v_{n x}=E \sin \phi / B$, which gives rise to the conventional Hall effect. The velocity component along the $y^{\prime}$ direction is zero.

Now, it is straightforward to calculate the transport coefficients in the rotated frame of reference, $z^{\prime}-y^{\prime}$. Using Eq. (29) to revert back to the laboratory frame, we find that the $B$-dependent part of the transport coefficients picks up the familiar angular dependence given by

$$
\begin{aligned}
& \left\{\sigma_{z z}, \alpha_{z z}, \bar{\alpha}_{z z}, \bar{\kappa}_{z z}\right\}=\left\{\sigma_{z z}^{\prime}, \alpha_{z z}^{\prime}, \bar{\alpha}_{z z}^{\prime}, \bar{\kappa}_{z z}^{\prime}\right\} \cos ^{2} \phi, \\
& \left\{\sigma_{y z}, \alpha_{y z}, \bar{\alpha}_{y z}, \bar{\kappa}_{y z}\right\}=\left\{\sigma_{z z}^{\prime}, \alpha_{z z}^{\prime}, \bar{\alpha}_{z z}^{\prime}, \bar{\kappa}_{z z}^{\prime}\right\} \sin \phi \cos \phi .
\end{aligned}
$$

In deriving Eqs. (31) and (32), we have used the fact that $L_{y y}^{\prime}=L_{y z}^{\prime}=0$. This follows from the fact that the velocity component along the $y^{\prime}$ axis is zero. This clearly establishes that the planar Hall transport coefficients retain the $B$ dependence of the longitudinal transport coefficients in the linear response regime. Consequently, they also display the three regimes of (i) ultraquantum transport which is linear in $B$, (ii) quantum oscillation transport regime, and (iii) the semiclassical transport regime which is quadratic in $B$.

The $B$ dependence of the longitudinal and the planar (Righi-Leduc) thermal conductivity is shown in Figs. 8(c) and 8(d), for different orientations of the magnetic field. The quantum oscillations in both of these can be clearly seen. Similar quantum oscillations will also be there in all other transport coefficients.

\section{DISCUSSION}

In this paper, we have presented all calculations within the constant scattering time approximation. The energy and temperature dependence of the scattering timescale can be easily included and it does not change the qualitative features of the discussed transport coefficients. However, we have to be more careful in analyzing the magnetic field dependence of the scattering timescale. For the short-range impurity scattering, realized via neutral defects, the scattering rate is proportional to the density of states $[8,53,60]$. Now, in the ultraquantum limit, the DOS is proportional to $B$, and hence $\tau_{v} \propto 1 / B$. Thus, the transport coefficients may become completely independent of the magnetic field in this regime. For the case of multiple filled LLs, the magnetic field dependence of the scattering timescale is more complicated. Additionally, the magnetic field dependence for other scattering mechanisms like charged impurities and phonons among others is still an open problem. For an isolated system, the chemical potential can also depend on the magnetic field strength. However in our case, the device is connected to external leads (or reservoirs), which set the chemical potential of the system [61].

As shown in Fig. 1, the semiclassical or quantum transport regimes are quantified by $\omega_{c} \tau$ or $\beta \hbar \omega_{c}$. Now, if $\omega_{c} \tau \gg 1$ along with $\beta \hbar \omega_{c} \gg 1$, then the LL broadening caused either by the impurities or by the thermal smearing is less than 
the LL separation, making them distinguishable. This is the regime where the discreteness of the LLs manifests itself in all physical quantities including transport coefficients. Here, the shortest scattering timescale dominates the disorder-induced broadening. This typically turns out to be the intranode scattering time $(\tau)$ in WMs. For a system with $v_{F}=2 \times 10^{5}$ $\mathrm{m} / \mathrm{s}$ and $\tau \approx 10^{-12} \mathrm{~s}$, we have $B \gg 0.02 \mathrm{~T}$ for observing quantized Landau levels in transport experiments. So a value of $B \approx 1 \mathrm{~T}$ is very likely to show the quantum oscillation regime. This corresponds to $\hbar \omega_{c} \approx 5 \mathrm{meV}$, which is easily much larger than the thermal energy scale for temperatures $T<60 \mathrm{~K}$. Furthermore, the use of Boltzmann transport equation assumes the presence of a weak disorder strength which preserves the shape of the Fermi surface. This is specified via the condition $\mu \gg \hbar / \tau$. Thus, to explore the ultraquantum limit, we have to be in the regime $\hbar / \tau<\mu<\hbar \omega_{c}$. For a magnetic field of $B=10 \mathrm{~T}$ and $\tau \approx 10^{-12} \mathrm{~s}$, this translates to $0.7 \mathrm{meV}<\mu<16 \mathrm{meV}$.

\section{CONCLUSIONS}

The origin of longitudinal magnetoresistance is physically very intriguing since the electrons do not feel any Lorentz force along the applied electric field. Particularly in chiral fluids such as WM, the longitudinal transport coefficients can also originate from CAs, which makes longitudinal transport in WM even more exciting $[62,63]$. In this paper, we have presented a unified framework for calculating the transport coefficients which captures all three transport regimes: (i) ultraquantum, (ii) quantum oscillation, and (iii) semiclassical. We derive explicit analytical expressions for all the CA-induced magnetotransport coefficients in the regime of multiple LL being occupied. We explicitly show that the mixed chiral-gravitational anomaly induced longitudinal thermal conductivity and the planar Righi-Leduc thermal conductivity display quantum oscillations with features similar to that in the longitudinal conductivity. Additionally, we find a linear- $B$ dependence of all magneto-transport coefficients in the ultraquantum limit, a quadratic- $B$ dependence in the semiclassical regime, and quantum oscillations in the intermediate regime. Our work will be useful in analyzing and interpreting the exciting magnetotransport experiments in Weyl metals.

\section{ACKNOWLEDGMENT}

We thank the Science Education and Research Board (SERB) and Department of Science and Technology (DST) of the Government of India, for funding support.

\section{APPENDIX A: EQUILIBRIUM CURRENT}

While the equilibrium current for each of the Weyl cone of a specific chirality is nonzero, the total contribution from two cones of opposite chirality add up to zero. In this Appendix, we outline the calculation of the equilibrium currents for each Weyl node. The equilibrium charge and energy currents are defined via the equation

$$
\left\{j_{e, \text { eq }}^{s}, j_{\mathcal{E}, \text { eq }}^{s}\right\}=\mathfrak{D} \sum_{n} \int \frac{d k_{z}}{2 \pi} v_{n z}^{s}\left\{-e, \epsilon_{n}^{s}\right\} f_{n}^{s} .
$$

Here, the integration limit is $k_{z} \in[-\infty, \infty]$. The contribution to both the currents from all LLs except for the $n=0$ level vanishes since the integrand is an odd function of $k_{z}$ for $n \neq 0$. A nonzero contribution arises only from the lowest LLs.

The $n=0$ LL for each chirality is linearly dispersing and extends to $\pm \infty$ in energy. For such systems, which have a vacuum state of infinite negative-energy states occupied, the physical currents have to be calculated by subtracting the contribution of the vacuum states. This is generally done by defining the current operators via normal ordering. We find that this is equivalent to adopting the integration limit of $k_{z} \in$ $[0, \infty)$ for $s=-1$ (or $k_{z} \in[-\infty, 0)$ for $s=1$ ) in Eq. (A1). Performing a variable change from $k_{z} \rightarrow \epsilon$, and doing an integration by parts, we obtain

$$
j_{\text {eq }}^{s} \propto\left[\left.g(\epsilon) f(\epsilon)\right|_{0} ^{\infty}-\int_{0}^{\infty} d \epsilon h(\epsilon) \frac{\partial f}{\partial \epsilon}\right] .
$$

Here, $g(\epsilon)$ and $h(\epsilon)$ are power functions of energy $\propto \epsilon^{n}$ with $n=1,2$ and the explicit form depends on whether we calculate the charge or energy current. It is straight forward to show that the first term in Eq. (A2) vanishes as $g(\epsilon) \rightarrow 0$ for $\epsilon \rightarrow 0$ and $f_{n}(\epsilon) \rightarrow 0$ for $\epsilon \rightarrow \infty$. The second term yields the expression of the equilibrium charge current [Eq. (4)] and the equilibrium energy current [Eq. (5)].

\section{APPENDIX B: CROSSED ELECTRIC AND MAGNETIC FIELDS}

The configuration of crossed fields where magnetic field is applied perpendicular to the bias voltage is common in the context of classical Hall effect. In this section, we solve the LL spectrum for such scenario. Let us consider the magnetic field to be $B \hat{\mathbf{z}}$ and electric field to be $E \hat{\mathbf{y}}$. The corresponding electromagnetic potentials we choose as $\mathbf{A}=(-B y, 0,0)$ and $\phi=-E y$. Now, the LL spectrum can be obtained by solving the wave equation $\hat{H} \Psi=\epsilon \Psi$ with

$$
\hat{H}=v_{F}\left[\sigma_{x}\left(\hat{p}_{x}-e B y\right)+\sigma_{y} \hat{p}_{y}+\sigma_{z} \hat{p}_{z}\right]+e E y \sigma_{0} .
$$

Here, $\sigma_{0}$ is a $2 \times 2$ identity matrix. Following Ref. [58], we can recast this wave equation in the four-momentum language with $p_{0}=\epsilon / v_{F}$ and make the Lorentz transformation as $[51,59]$

$$
\left(\begin{array}{l}
\hat{p}_{0} \\
\hat{p}_{1}
\end{array}\right)=\left(\begin{array}{cc}
\cosh \theta & \sinh \theta \\
\sinh \theta & \cosh \theta
\end{array}\right)\left(\begin{array}{c}
\hat{\tilde{p}}_{0} \\
\hat{\tilde{p}}_{1}
\end{array}\right) .
$$

Here, $\tanh \theta=\frac{E}{v_{F} B}$. This Lorentz transformation takes us to a frame which moves along the positive $x$ direction with velocity $v_{F} \tanh \theta$. In this frame, there is no electric field. Now, using the identities $\sigma_{x}^{2}=\sigma_{0}$ and $\left(\sigma_{0} \cosh \theta-\sigma_{x} \sinh \theta\right)=$ $\exp \left(-\theta \sigma_{x}\right)$, we rewrite the wave equation as

$$
v_{F}\left[\sigma_{x}\left(\hat{\tilde{p}}_{x}-\frac{e B}{\cosh \theta} \tilde{y}\right)+\sigma_{y} \hat{\tilde{p}}_{2}+\sigma_{z} \hat{\tilde{p}}_{z}-\sigma_{0} \hat{\tilde{p}}_{0}\right] \tilde{\Psi}=0 .
$$

Here, $\tilde{\Psi}(\tilde{t}, \tilde{x}, \tilde{y}, \tilde{z})=\exp \left(-\sigma_{x} \theta / 2\right) \Psi(t, x, y, z)$. Equation (B3) represents the familiar wave equation in the presence of a magnetic field only and its eigenvalue and wave functions are known. The only difference is the strength of the magnetic field is modified as $B \rightarrow B / \cosh \theta$. Using $\tilde{B}=B / \cosh \theta$ and 
$s_{n}=\operatorname{sgn}(n)$, the LL spectrum is obtained to be

$$
\tilde{\epsilon}_{n}\left(\tilde{p}_{z}\right)=s_{n} \sqrt{\left(v_{F} \tilde{p}_{z}\right)^{2}+2|n| \hbar e \tilde{B} v_{F}^{2}}-s v_{F} \tilde{p}_{z} \delta_{n, 0} .
$$

After doing the inverse Lorentz transformation, we obtain

$$
\epsilon_{n}^{s}\left(k_{x}, k_{z}\right)=\frac{1}{\eta^{2}} \hbar v_{F} \mathcal{K}_{n}^{s}\left(k_{z}\right)+\hbar v_{F} k_{x} \tanh \theta .
$$

Here, we have defined $\eta^{-2}=\sqrt{1-\tanh ^{2} \theta}$ and

$$
\mathcal{K}_{n}^{s}\left(k_{z}\right)=\operatorname{sgn}(n) \sqrt{\frac{2|n|}{\left(l_{B} \eta\right)^{2}}+k_{z}^{2}}-s k_{z} \delta_{n, 0} .
$$

The most notable effect of the perpendicular electric field is the second term in Eq. (B5). As a result of this, the carriers have finite velocity along the $x$ axis, perpendicular to the $\mathbf{E}-\mathbf{B}$ plane. It is straightforward to calculate

$$
v_{n z}=\frac{v_{F}}{\eta^{2}}\left(s_{n} \frac{k_{z}}{\sqrt{\frac{2 n}{\tilde{I}_{B}^{2}}+k_{z}^{2}}}-s \delta_{n, 0}\right) ; \quad v_{n x}=\frac{E}{B} \text {. }
$$

We note that the $x$ component of the velocity is simply the Lorentz velocity, which is a constant and identical for all the LLs. This is what gives rise to the classical Hall effect.

We emphasize that Eq. (B3) represents a harmonic oscillator with center at $y=\left(\tilde{l}_{B}\right)^{2} \tilde{p}_{x} / \hbar$. In the laboratory frame, this can be written as

$$
y_{c}^{\prime}=l_{B}^{2} k x+l_{B}^{2} \mathcal{K}_{n}^{s}\left(k_{z}\right) \sinh \theta .
$$

The perpendicular electric field in this expression lifts the degeneracy of LLs, and consequently it modifies the DOS. Using the general formula of DOS, $\rho(\epsilon)=\sum_{n=0}^{n_{c}} \frac{1}{(2 \pi)^{2}}$ $\iint d k_{x} d k_{z} \delta\left(\epsilon_{n}^{s}-\epsilon\right)$, a small calculation yields

$$
\rho(\epsilon)=\eta^{2} \rho_{0}\left[2 \sum_{n=0}^{n_{c}} \frac{\lambda_{n}(\epsilon,+)-\lambda_{n}(\epsilon,-)}{e E L_{y}}-1\right] .
$$

Here, we have defined

$$
\lambda_{n}(\epsilon, \pm)=\sqrt{\left(\epsilon \pm \frac{e E L_{y}}{2}\right)^{2}-2|n|\left(\hbar \omega_{c} \eta^{-3}\right)^{2}} .
$$

Equation (B9) is a nonlinear function of the electric field strength.

In the limit of $e E L_{y} \ll \epsilon$, expanding Eq. (B10) in powers of the strength of the electric field we obtain the DOS to be $\rho(\epsilon)=\eta^{2} \rho_{0} \Theta_{0}$. Here,

$$
\Theta_{0}=2 \sum_{n=0}^{n_{c}} \frac{1}{\sqrt{1-2|n|\left(\hbar \omega_{c} \eta^{-3}\right)^{2}}}-1 .
$$

This expression of the DOS was used in Ref. [51] to calculate the angular dependence of the planar Hall conductivity. However, in this paper, we will focus on the linear response regime and retain only the term independent of the electric field. To the lowest order in $E$, we have $\eta \rightarrow 1$, and Eq. (B9) reduces to the expression of the DOS derived in Eq. (21), which was independent of $E$.
[1] X. Wan, A. M. Turner, A. Vishwanath, and S. Y. Savrasov, Topological semimetal and fermi-arc surface states in the electronic structure of pyrochlore iridates, Phys. Rev. B 83, 205101 (2011).

[2] A. A. Burkov and L. Balents, Weyl Semimetal in a Topological Insulator Multilayer, Phys. Rev. Lett. 107, 127205 (2011).

[3] N. P. Armitage, E. J. Mele, and A. Vishwanath, Weyl and Dirac semimetals in three-dimensional solids, Rev. Mod. Phys. 90, 015001 (2018).

[4] H.-J. Kim, K.-S. Kim, J.-F. Wang, M. Sasaki, N. Satoh, A. Ohnishi, M. Kitaura, M. Yang, and L. Li, Dirac Versus Weyl Fermions in Topological Insulators: Adler-Bell-Jackiw Anomaly in Transport Phenomena, Phys. Rev. Lett. 111, 246603 (2013).

[5] S. A. Yang, H. Pan, and F. Zhang, Dirac and Weyl Superconductors in Three Dimensions, Phys. Rev. Lett. 113, 046401 (2014).

[6] A. A. Burkov, Chiral Anomaly and Diffusive Magnetotransport in Weyl Metals, Phys. Rev. Lett. 113, 247203 (2014).

[7] S. A. Parameswaran, T. Grover, D. A. Abanin, D. A. Pesin, and A. Vishwanath, Probing the Chiral Anomaly with Nonlocal Transport in Three-Dimensional Topological Semimetals, Phys. Rev. X 4, 031035 (2014).

[8] A. A. Burkov, Negative longitudinal magnetoresistance in Dirac and Weyl metals, Phys. Rev. B 91, 245157 (2015).
[9] A. Cortijo, Y. Ferreirós, K. Landsteiner, and M. A. H. Vozmediano, Elastic Gauge Fields in Weyl Semimetals, Phys. Rev. Lett. 115, 177202 (2015).

[10] M. N. Chernodub, A. Cortijo, and M. A. H. Vozmediano, Generation of a Nernst Current from the Conformal Anomaly in Dirac and Weyl Semimetals, Phys. Rev. Lett. 120, 206601 (2018).

[11] Z. Song and X. Dai, Hear the Sound of Weyl Fermions, Phys. Rev. X 9, 021053 (2019).

[12] J. Xiang, S. Hu, Z. Song, M. Lv, J. Zhang, L. Zhao, W. Li, Z. Chen, S. Zhang, J.-T. Wang, Y.-f. Yang, X. Dai, F. Steglich, G. Chen, and P. Sun, Giant Magnetic Quantum Oscillations in the Thermal Conductivity of TaAs: Indications of Chiral Zero Sound, Phys. Rev. X 9, 031036 (2019).

[13] K. Sonowal, A. Singh, and A. Agarwal, Giant optical activity and Kerr effect in type-I and type-II Weyl semimetals, Phys. Rev. B 100, 085436 (2019).

[14] C. Wang, L. Gioia, and A. A. Burkov, Fractional Quantum Hall Effect in Weyl Semimetals, Phys. Rev. Lett. 124, 096603 (2020).

[15] K. Sadhukhan, A. Politano, and A. Agarwal, Novel Undamped Gapless Plasmon Mode in a Tilted Type-II Dirac Semimetal, Phys. Rev. Lett. 124, 046803 (2020).

[16] S. L. Adler, Axial-vector vertex in spinor electrodynamics, Phys. Rev. 177, 2426 (1969). 
[17] J. S. Bell and R. Jackiw, A PCAC puzzle: $\pi^{0} \rightarrow \gamma \gamma$ in the $\sigma$ model, Nuovo Cimento A (1965-1970) 60, 47 (1969).

[18] H. B. Nielsen and M. Ninomiya, The Adler-Bell-Jackiw anomaly and Weyl fermions in a crystal, Phys. Lett. B 130, 389 (1983).

[19] K. Landsteiner, Notes on anomaly induced transport, Acta Phys. Pol. B 47, 2617 (2016).

[20] The terms Weyl metal and Weyl semimetal are used on the same footing in the literature. However, materials hosting Weyl fermions with a finite density of states at the Fermi energy should be termed as Weyl metals, and Weyl semimetals should be used only for the case of vanishing density of states at the Fermi energy.

[21] J. Xiong, S. K. Kushwaha, T. Liang, J. W. Krizan, M. Hirschberger, W. Wang, R. J. Cava, and N. P. Ong, Evidence for the chiral anomaly in the Dirac semimetal $\mathrm{Na}_{3} \mathrm{Bi}$, Science 350, 413 (2015).

[22] S.-M. Huang, S.-Y. Xu, I. Belopolski, C.-C. Lee, G. Chang, B. Wang, N. Alidoust, G. Bian, M. Neupane, C. Zhang, S. Jia, A. Bansil, H. Lin, and M. Zahid Hasan, A Weyl fermion semimetal with surface Fermi arcs in the transition metal monopnictide TaAs class, Nat. Commun. 6, 7373 (2015).

[23] H. Li, H. He, H.-Z. Lu, H. Zhang, H. Liu, R. Ma, Z. Fan, S.-Q. Shen, and J. Wang, Negative magnetoresistance in Dirac semimetal $\mathrm{Cd}_{3} \mathrm{As}_{2}$, Nat. Commun. 7, 10301 (2016).

[24] C.-L. Zhang, S.-Y. Xu, I. Belopolski, Z. Yuan, Z. Lin, B. Tong, G. Bian, N. Alidoust, C.-C. Lee, S.-M. Huang, T.-R. Chang, G. Chang, C.-H. Hsu, H.-T. Jeng, M. Neupane, D. S. Sanchez, H. Zheng, J. Wang, H. Lin, C. Zhang, H.-Z. Lu, S.-Q. Shen, T. Neupert, M. Zahid Hasan, and S. Jia, Signatures of the AdlerBell-Jackiw chiral anomaly in a Weyl fermion semimetal, Nat. Commun. 7, 10735 (2016).

[25] K. Landsteiner, E. Megías, and F. Pena-Benitez, Gravitational Anomaly and Transport Phenomena, Phys. Rev. Lett. 107, 021601 (2011).

[26] A. Lucas, R. A. Davison, and S. Sachdev, Hydrodynamic theory of thermoelectric transport and negative magnetoresistance in Weyl semimetals, Proc. Natl. Acad. Sci. USA 113, 9463 (2016).

[27] J. Gooth, A. C. Niemann, T. Meng, A. G. Grushin, K. Landsteiner, B. Gotsmann, F. Menges, M. Schmidt, C. Shekhar, V. Süß, R. Hühne, B. Rellinghaus, C. Felser, B. Yan, and K. Nielsch, Experimental signatures of the mixed axialgravitational anomaly in the Weyl semimetal NBP, Nature (London) 547, 324 (2017).

[28] M. Stone and J. Kim, Mixed anomalies: Chiral vortical effect and the sommerfeld expansion, Phys. Rev. D 98, 025012 (2018).

[29] K. Das and A. Agarwal, Thermal and gravitational chiral anomaly induced magneto-transport in Weyl semimetals, Phys. Rev. Research 2, 013088 (2020).

[30] M. Hirschberger, S. Kushwaha, Z. Wang, Q. Gibson, S. Liang, C. A. Belvin, B. A. Bernevig, R. J. Cava, and N. P. Ong, The chiral anomaly and thermopower of weyl fermions in the halfHeusler GdPtBi, Nat. Mater. 15, 1161 (2016).

[31] Z. Jia, C. Li, X. Li, J. Shi, Z. Liao, D. Yu, and X. Wu, Thermoelectric signature of the chiral anomaly in $\mathrm{Cd}_{3} \mathrm{As}_{2}$, Nat. Commun. 7, 13013 (2016).

[32] A. A. Burkov, Giant planar Hall effect in topological metals, Phys. Rev. B 96, 041110(R) (2017)
[33] S. Nandy, G. Sharma, A. Taraphder, and S. Tewari, Chiral Anomaly as the Origin of the Planar Hall Effect in Weyl Semimetals, Phys. Rev. Lett. 119, 176804 (2017).

[34] K. Das and A. Agarwal, Berry curvature induced thermopower in type-I and type-II Weyl semimetals, Phys. Rev. B 100, 085406 (2019).

[35] G. Sharma and S. Tewari, Transverse thermopower in Dirac and Weyl semimetals, Phys. Rev. B 100, 195113 (2019).

[36] S. Nandy, A. Taraphder, and S. Tewari, Planar thermal Hall effect in Weyl semimetals, Phys. Rev. B 100, 115139 (2019).

[37] H. Li, H.-W. Wang, H. He, J. Wang, and S.-Q. Shen, Giant anisotropic magnetoresistance and planar Hall effect in the Dirac semimetal $\mathrm{Cd}_{3} \mathrm{As}_{2}$, Phys. Rev. B 97, 201110(R) (2018).

[38] N. Kumar, S. N. Guin, C. Felser, and C. Shekhar, Planar Hall effect in the Weyl semimetal GdPtBi, Phys. Rev. B 98, 041103(R) (2018).

[39] P. Li, C. H. Zhang, J. W. Zhang, Y. Wen, and X. X. Zhang, Giant planar Hall effect in the Dirac semimetal $\mathrm{ZrTe}_{5-\delta}$, Phys. Rev. B 98, 121108(R) (2018).

[40] J. Yang, W. L. Zhen, D. D. Liang, Y. J. Wang, X. Yan, S. R Weng, J. R. Wang, W. Tong, L. Pi, W. K. Zhu, and C. J. Zhang, Current jetting distorted planar Hall effect in a Weyl semimetal with ultrahigh mobility, Phys. Rev. Mater. 3, 014201 (2019).

[41] D. T. Son and B. Z. Spivak, Chiral anomaly and classical negative magnetoresistance of Weyl metals, Phys. Rev. B 88 104412 (2013).

[42] K.-S. Kim, H.-J. Kim, and M. Sasaki, Boltzmann equation approach to anomalous transport in a Weyl metal, Phys. Rev. B 89, 195137 (2014).

[43] S.-K. Yip, Kinetic equation and magneto-conductance for Weyl metal in the clean limit, arXiv:1508.01010.

[44] K. Das and A. Agarwal, Linear magnetochiral transport in tilted type-I and type-II Weyl semimetals, Phys. Rev. B 99, 085405 (2019).

[45] R. Lundgren, P. Laurell, and G. A. Fiete, Thermoelectric properties of Weyl and Dirac semimetals, Phys. Rev. B 90, 165115 (2014).

[46] K.-S. Kim, Role of axion electrodynamics in a Weyl metal: Violation of Wiedemann-Franz law, Phys. Rev. B 90, 121108(R) (2014).

[47] B. Z. Spivak and A. V. Andreev, Magnetotransport phenomena related to the chiral anomaly in Weyl semimetals, Phys. Rev. B 93, 085107 (2016).

[48] G. Sharma, P. Goswami, and S. Tewari, Nernst and magnetothermal conductivity in a lattice model of Weyl fermions, Phys. Rev. B 93, 035116 (2016).

[49] E. V. Gorbar, V. A. Miransky, and I. A. Shovkovy, Chiral anomaly, dimensional reduction, and magnetoresistivity of Weyl and Dirac semimetals, Phys. Rev. B 89, 085126 (2014).

[50] M.-X. Deng, G. Y. Qi, R. Ma, R. Shen, R.-Q. Wang, L. Sheng, and D. Y. Xing, Quantum Oscillations of the Positive Longitudinal Magnetoconductivity: A Fingerprint for Identifying Weyl Semimetals, Phys. Rev. Lett. 122, 036601 (2019).

[51] M.-X. Deng, H.-J. Duan, W. Luo, W. Y. Deng, R.-Q. Wang, and L. Sheng, Quantum oscillation modulated angular dependence of the positive longitudinal magnetoconductivity and planar Hall effect in Weyl semimetals, Phys. Rev. B 99, 165146 (2019).

[52] C. Schindler, S. Galeski, W. Schnelle, R. Wawrzyńczak, W. Abdel-Haq, S. N. Guin, J. Kroder, N. Kumar, C. Fu, H. Borrmann, C. Shekhar, C. Felser, T. Meng, A. G. Grushin, 
Y. Zhang, Y. Sun, and J. Gooth, Anisotropic electrical and thermal magnetotransport in the magnetic semimetal GdPtBi, Phys. Rev. B 101, 125119 (2020).

[53] V. Aji, Adler-Bell-Jackiw anomaly in Weyl semimetals: Application to pyrochlore iridates, Phys. Rev. B 85, 241101(R) (2012).

[54] S.-B. Zhang, H.-Z. Lu, and S.-Q. Shen, Linear magnetoconductivity in an intrinsic topological Weyl semimetal, New J. Phys. 18, 053039 (2016).

[55] D. Vu, W. Zhang, C. Şahin, M. Flatté, N. Trivedi, and J. P. Heremans, Thermal chiral anomaly in the magnetic-field induced ideal Weyl phase of $\mathrm{Bi}_{1}-\mathrm{xSb}_{x}$ topological insulators, arXiv:1906.02248.

[56] These quantum oscillations are similar in nature to the Subhkinov-de Haas oscillations although their origin is different. Both these oscillations arise from the depopulation of the Landau levels as they cross the Fermi energy, and both these oscillation are periodic in $1 / B$.

[57] N. W. Ashcroft and N. D. Mermin, Solid State Physics (Holt, Rinehart and Winston, New York, 1976).
[58] V. Lukose, R. Shankar, and G. Baskaran, Novel Electric Field Effects on Landau Levels in Graphene, Phys. Rev. Lett. 98, 116802 (2007).

[59] Z. Z. Alisultanov, Effect of a transverse electric field on the Landau bands in a Weyl semimetal, JETP Lett. 105, 442 (2017).

[60] H.-Z. Lu and S.-Q. Shen, Quantum transport in topological semimetals under magnetic fields, Front. Phys. 12, 127201 (2017).

[61] A. N. Roy Choudhury and V. Venkataraman, Measuring the magnetic-field-dependent chemical potential of a lowdensity three-dimensional electron gas in $\mathbf{n}$-GaAs and extracting its magnetic susceptibility, Phys. Rev. B 93, 045208 (2016).

[62] H. K. Pal and D. L. Maslov, Necessary and sufficient condition for longitudinal magnetoresistance, Phys. Rev. B 81, 214438 (2010).

[63] Y. Gao, S. A. Yang, and Q. Niu, Intrinsic relative magnetoconductivity of nonmagnetic metals, Phys. Rev. B 95, 165135 (2017). 